\title{
Correlação entre condições de saneamento básico e parasitoses intestinais na população de Assis, Estado de São Paulo
}

\author{
Correlation between sanitation conditions and enteroparasitoses \\ in the population of Assis, São Paulo State, Brazil
}

\author{
Karin Maria Ludwig, Fernando Frei, Firmino Alvares Filho \\ e João Tadeu Ribeiro-Paes
}

\begin{abstract}
Resumo Foi estudada a distribuição dos enteroparasitos mais freqüentes na população de Assis, São Paulo, de 1990 a 1992. Foram analisados 18.366 exames oriundos de seis postos de atendimento sanitário (PAS): Vila Marialves, Vila Progresso, Centro, Vila Xavier, Vila Fiúza e Vila Bonfim. A prevalência de enteroparasitoses geral foi $23,3 \%$. Os enteroparasitos mais encontrados foram: Giardia intestinalis (8,7\%), Ascaris lumbricoides (5,5\%), Trichuris trichiura $(2,4 \%) e$ Hymenolepis nana (1,9\%). Na Vila Marialves, região de baixo nível sócio-econômico essas freqüências foram, respectivamente: 17\%; 13,1\%; 5,9\% e 4,2\%. A faixa etária 3 a 12 anos apresentou maior número de indivíduos parasitados. Estabeleceu-se uma correlação entre as condições de saneamento básico, expressos pelo número de ligações de água e esgoto, e a freqüểncia de parasitoses. Houve queda na freqüência de parasitoses nos PAS entre 1990 e 1992, coincidindo com o aumento do número de ligações de água e esgoto nestas regiões.
\end{abstract}

Palavras-chaves: Enteroparasitoses. Prevalência. Saneamento básico. Epidemiologia.

\begin{abstract}
The distribution of the most frequent enteroparasites in the population of Assis, State of São Paulo, was studied from 1990 to 1992. A total of 18,366 medical examinations from six sanitary care centers in the neighbourhoods of Marialves, Progresso, city center, Xavier, Fiúza and Bonfim were analized. The general prevalence of enteroparasites was $23.3 \%$. The most frequently found enteroparasites were: Giardia intestinalis (8.7\%), Ascaris lumbricoides (5.5\%), Trichuris trichiura (2.4\%) and Hymenolepis nana (1.9\%). In Marialves, a low income neighborhood, the prevalences were: 17\%; 13.1\%; 5.9\% and 4.2\%, rspectively. The age group from 3 to 12 years showed the largest number of infected individuals. There was a correlation between basic sanitation conditions, expressed as the number of places connected to the city water and sewage systems, and the prevalence of parasites. There was also a decrease of parasite prevalence in all sanitary care centers from 1990 to 1992, which coincided with the increase in the number of new water and sewage systems in these neighborhoods.
\end{abstract}

Key-words: Enteroparasitosis. Prevalence. Sanitation. Epidemiology.

Departamentos de Ciências Biológicas e Psicologia Experimental e do Trabalho da Universidade Estadual Paulista, Assis, SP, Brasil. Endereço para correspondência: Prof. João Tadeu Ribeiro-Paes, Depto. de Ciências Biológicas/UNESP, Av. Dom Antonio 2100, Caixa Postal 335, 19800-000 Assis, SP.

E-mail: jtrpaes@assis.unesp.br

Recebido para publicação em 21/7/98. 
As parasitoses intestinais constituem-se num grave problema de saúde pública, sobretudo nos países do terceiro mundo, sendo um dos principais fatores debilitantes da população, associando-se freqüentemente a quadros de diarréia crônica e desnutrição, comprometendo, como conseqüência, o desenvolvimento físico e intelectual, particularmente das faixas etárias mais jovens da população ${ }^{14} 1517$.

Em decorrência dos efeitos deletérios à saúde dos indivíduos e sobretudo, das repercussões econômicas, vários programas tem sido dirigidos para o controle das parasitoses intestinais em diferentes países, mas, infelizmente, constatase um descompasso entre o êxito alcançado nos países mais desenvolvidos e aquele verificado nas economias mais pobres. Além do custo financeiro das medidas técnicas, a falta de projetos educativos com a participação da comunidade dificultam a implementação das ações de controle. Há que se considerar, portanto, que além da melhoria das condições sócio-econômicas e de infra-estrutura geral, o engajamento comunitário é um dos aspectos fundamentais para a implantação, desenvolvimento e sucesso dos programas de controle ${ }^{51317}$.

Está bem estabelecido que as parasitoses intestinais são mais freqüentes em regiões menos desenvolvidas, considerado o sentido mais amplo da palavra ${ }^{16}$. Nos países subdesenvolvidos as parasitoses intestinais atingem índices de até $90 \%$, ocorrendo um aumento significativo da freqüência à medida que piora o nível socioeconômico ${ }^{19}$. Pesquisas populacionais sobre parasitos intestinais foram realizados em diversas regiões do Brasil e mostram freqüências bastante diferentes, de acordo com as condições locais de saneamento e características da amostra analisada 34589111213151617 .

No presente trabalho, procurou-se dimensionar alguns parâmetros epidemiológicos das principais enteroparasitoses em indivíduos da cidade de Assis, SP de acordo com o local de residência e faixa etária, bem como correlacionar as freqüências encontradas com as condições de saneamento básico das diferentes regiões da cidade.

\section{MATERIAL E MÉTODOS}

Foram analisados os resultados de 18.366 exames parasitológicos de fezes, realizados pelo Centro de Saúde de Assis, Estado de São Paulo, durante o período de 1990 a 1992 . O material desses exames foi recebido pelos seis postos de atendimento sanitário (PAS): Vila Marialves, Vila Progresso, CSI-Centro, CSIII-Vila Xavier, Vila Fiúza e Vila Bonfim, cuja localização e bairros

beneficiados de cada uma dessas regiões da cidade de Assis se encontra na Figura 1. Estas amostras eram recebidas aleatoriamente, de acordo com os pedidos médicos, o que quer dizer que os pacientes que procuravam os PAS não apresentavam exclusivamente queixas relacionadas a parasitoses intestinais.

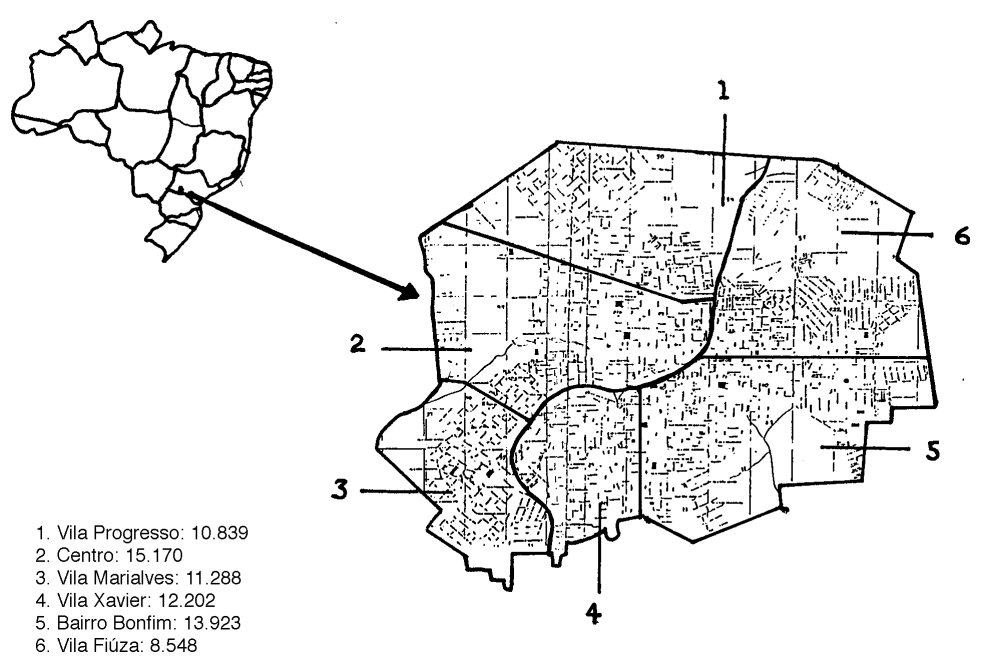

Figura 1 - A cidade de Assis no Brasil. Localização dos bairros e população atendida pelos Postos de Atendimento Sanitário (PAS). 
Todas as amostras analisadas foram submetidas aos exames coproparasitológicos de sedimentação espont,nea ${ }^{7}$.

Os dados de ligações de água e esgoto, como indicadores de saneamento básico, foram obtidos junto à SABESP - Companhia Paulista de Saneamento Básico, regional de Assis e se encontram na Figura 2, enquanto a população de cada uma destas regiões atendidas pelos Postos de Atendimento Sanitário (PAS) foi obtida junto ao IBGE (Instituto Brasileiro de Geografia e Estatística) - Assis e pode ser encontrada na Figura 1, de acordo com a sua localização na cidade de Assis.

A partir dos resultados dos exames foram calculadas as prevalências dos parasitos intestinais

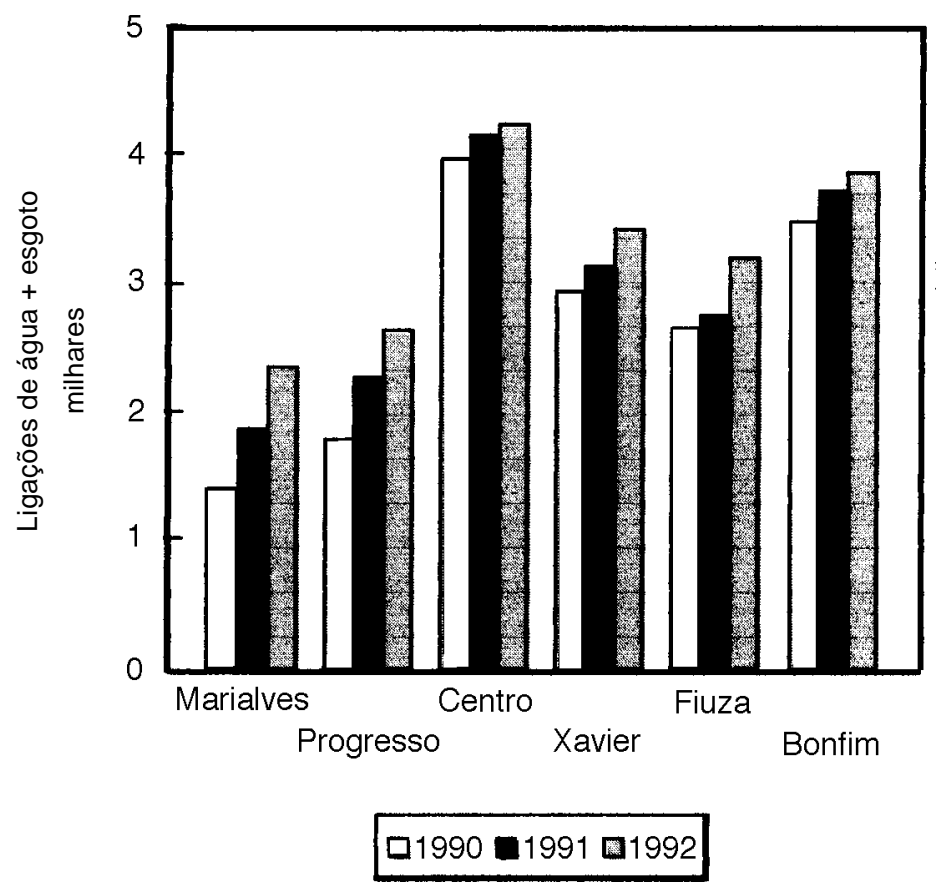

Figura 2 - Ligações de água e esgoto por região atendida pelo PAS de Assis. Fonte: SABESP:Companhia Palista de Saneamento Básico, regional de Assis.

encontrados e a distribuição dessas prevalências foi analisada quanto a faixa etária em intervalos de 3 anos.

A análise dos dados obtidos foi feita em três etapas: a) estudo descritivo das seis regiões assistidas pelos postos de atendimento sanitário e da cidade de Assis como um todo; b) verificação da hipótese de diferenciabilidade nos PAS e nas faixas etárias, quanto à prevalência parasitológica; c) análise de correlação entre a freqüência parasitológica e as condições de saneamento básico.
Assis localiza-se na região oeste do Estado de São Paulo, sendo o centro da cidade situado entre $22^{\circ} 40^{\prime}$ paralelo e $50^{\circ} 25^{\prime}$ meridiano. O clima da região é temperado brando, com inverno seco e verão quente e chuvoso. Apresenta um índice pluviométrico de $1250 \mathrm{~mm} / \mathrm{ano}$ e a temperatura média anual é de $22^{\circ} \mathrm{C}$, com a média no mês mais quente (janeiro) entre 24 e $25^{\circ} \mathrm{C}$ e do mês mais frio (julho) entre 17 e $18^{\circ} \mathrm{C}$.

\section{RESULTADOS}

Foram analisados exames coproparasitológicos de 18.366 indivíduos, nos períodos de 1990 a 1992, e o percentual de parasitos intestinais na amostra estudada foi de $23,3 \%$. Os resultados evidenciaram também que $12,2 \%$ dos indivíduos apresentaram protozoários não patogênicos como: Entamoeba coli (9\%), Endolimax nana (2,9\%) e lodamoeba butschilii $(0,3 \%)$. 
Quanto à distribuição total de enteroparasitos na população estudada (Figura 3) observa-se uma tendência ao decréscimo progressivo na prevalência de protozoários e helmintos, evidenciando-se esta queda a partir de 1991. Verifica-se, ainda, pela análise da Figura 2, uma diminuição mais expressiva da prevalência para os helmintos com um decréscimo de aproximadamente $60 \%$ de 1990 a 1992.
Considerando a distribuição dos parasitos nas regiões servidas pelos PAS, verificamos que as freqüências variam, inclusive dentro de um mesmo posto de atendimento sanitário ao longo do período analisado (Figura 4). A distribuição total de cada tipo de enteroparasito nos PAS é indicada na Tabela 1. $\mathrm{Na}$ amostra analisada merecem destaque, como enteroparasitos mais encontrados nos seis PAS, Giardia

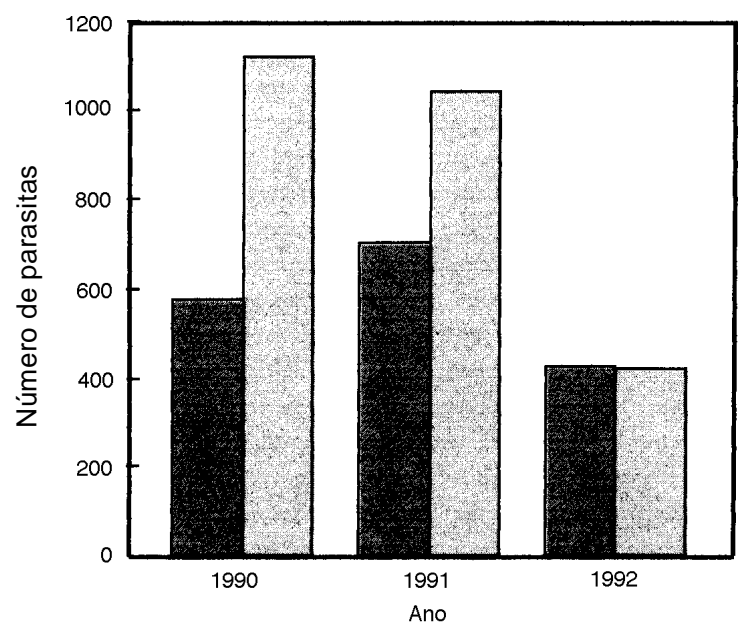

䊅 protozoários $\quad$ helmintos

Figura 3 - Distribuição geral de protozoários e helmintos encontrados nos exames de fezes.

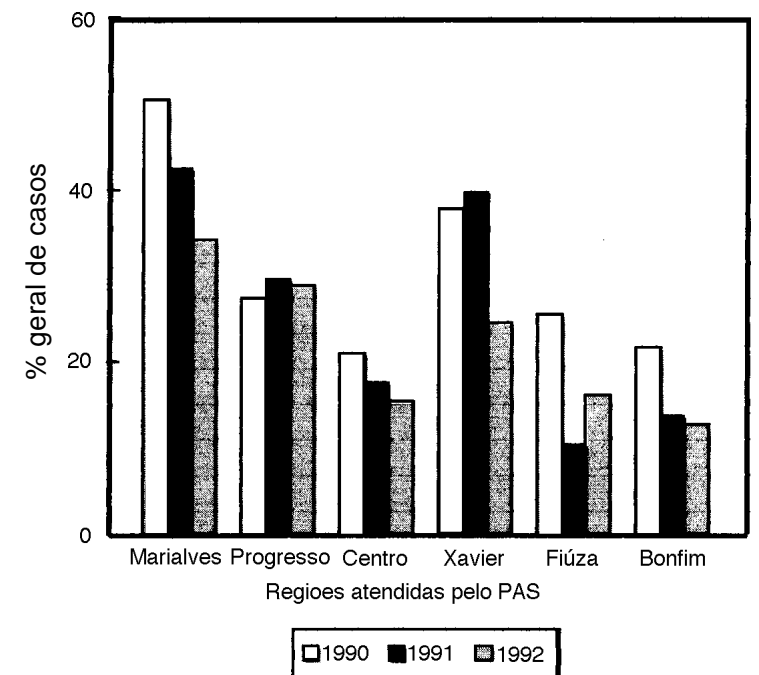

Figura 4 - Porcentagem de casos positivos dos parasitos intestinais encontrados nos PAS da cidade de Assis nos 3 anos de estudo (19901991-1992). 
Tabela 1 - Distribuição dos parasitos intestinais, por porcentagem de casos positivos, encontrados nos PAS durante os 3 anos de estudo (1990/1991/1992).

\begin{tabular}{lcccccccccc}
\hline PAS & Eh & $\mathrm{Gl}$ & $\mathrm{Al}$ & $\mathrm{Tt}$ & $\mathrm{A}$ & $\mathrm{Sm}$ & $\mathrm{Hn}$ & $\mathrm{Ev}$ & $\mathrm{T}$ & $\mathrm{Ss}$ \\
\hline Marialves & 0,3 & 17,0 & 13,1 & 5,9 & 1,6 & 0,2 & 4,2 & 0,4 & 0,1 & 2,2 \\
Progresso & 0,2 & 10,4 & 6,1 & 2,9 & 1,5 & - & 2,8 & 0,5 & 0,1 & 3,9 \\
CSI & 0,6 & 6,6 & 3,8 & 1,6 & 1,5 & 0,1 & 1,2 & 0,3 & 0,5 & 1,5 \\
CSIII & 0,9 & 15,6 & 8,9 & 4,8 & 1,5 & 0,2 & 2,1 & 0,2 & 0,2 & 2,8 \\
Fiúza & - & 7,2 & 4,3 & 1,8 & 1,8 & 0,4 & 1,9 & 0,2 & - & 1,9 \\
Bonfim & 0,5 & 5,8 & 3,7 & 1,7 & 0,9 & - & 1,4 & 0,4 & 0,5 & 1,2 \\
\hline Total & 0,5 & 8,7 & 5,5 & 2,4 & 1,5 & 0,1 & 1,9 & 0,3 & 0,4 & 1,9 \\
\hline
\end{tabular}

Eh. Entamoeba histolytica; Gl. Giardia intestinalis; AL. Ascaris lumbricoides; Tt. Trichuris trichiura; A. ancylostomatidae; Sm. Schistosoma mansoni; Hn. Hymenolepis nana; Ev. Enterobius vermicularis; T. Taenia sp; Ss. Strongyloides stercoralis.

intestinalis (8,7\%), Ascaris lumbricoides (5,5\%) e Trichuris trichiura (2,4\%).

A partir da Figura 4 e Tabela 2, pode-se verificar que no final dos três anos analisados, as freqüências mais elevadas de enteroparasitos foram encontradas nas populações que utilizam os PAS da Vila Marialves (45,1\%), Vila Progresso $(28,6 \%)$ e CSIII- Vila Xavier (37,3\%), que são regiões periféricas da cidade e que abrangem as populações de nível sócio-econômico mais baixo, enquanto freqüências menores foram encontradas nas populações dos PAS do Bairro Bonfim $(16,2 \%)$ e CSI-Centro (17,6\%) que englobam as populações com nível sócio-econômico melhor que o da população anteriormente mencionada. Sendo que na Vila Fiúza, observa-se uma

Tabela 2 - Freqüências gerais dos parasitos intestinais encontrados nos PAS da cidade de Assis durante os 3 anos de estudo (1990/1991/1992).

\begin{tabular}{|c|c|c|c|c|c|c|c|c|c|c|}
\hline \multirow[b]{2}{*}{ PAS } & \multicolumn{3}{|c|}{1990} & \multicolumn{3}{|c|}{1991} & \multicolumn{3}{|c|}{1992} & \multirow{2}{*}{$\begin{array}{c}3 \text { anos } \\
\%\end{array}$} \\
\hline & amostra & casos & $\%$ & amostra & casos & $\%$ & amostra & casos & $\%$ & \\
\hline Marialves & 1105 & 558 & 50,5 & 1106 & 480 & 43,4 & 374 & 128 & 34,2 & 45,1 \\
\hline Progresso & 855 & 234 & 27,4 & 1011 & 300 & 29,7 & 132 & 38 & 28,7 & 28,6 \\
\hline CSI & 3024 & 634 & 20,9 & 4337 & 761 & 17,5 & 4212 & 641 & 15,2 & 17,6 \\
\hline CSIII & 294 & 111 & 37,7 & 252 & 100 & 39,7 & 61 & 15 & 24,6 & 37,2 \\
\hline Fiúza & 290 & 74 & 25,5 & 158 & 16 & 10,1 & 62 & 10 & 16,1 & 19,6 \\
\hline Bonfim & 377 & 81 & 21,5 & 605 & 82 & 13,5 & 111 & 14 & 12,6 & 16,2 \\
\hline Total & 5945 & 1692 & 28,5 & 7469 & 1739 & 23,3 & 4952 & 846 & 17,08 & 23,3 \\
\hline
\end{tabular}

porcentagem de casos positivos de aproximadamente $20 \%$, bairro onde se encontrava um conjunto habitacional em formação, recebendo famílias provenientes de diferentes regiões da cidade.

Observando-se ainda a Figura 4, podemos verificar que, de um modo geral, houve um decréscimo nos casos positivos de enteroparasitoses intestinais entre 1990 e 1992, o que coincide com o aumento das ligações de água e esgoto em todas as regiões da cidade de Assis, como pode ser observado nas Figura 2 e Tabela 4.

Quanto à freqüência de enteroparasitos observadas em diferentes faixas etárias durante o período de estudo (Figura 5), verifica-se que na faixa etária de 3 a 6 anos encontram-se as freqüências mais elevadas (38,2\%). Na faixa de
6 a 9 anos incidência continua elevada $(32,7 \%)$, porém com tendência ao decréscimo que se mantém com o aumento da faixa etária, verificando-se freqüências relativamente baixas $(12,3 \%)$ para os maiores de 18 anos. Para todas as faixas etárias enteroparasitos mais encontrados foram Giardia intestinalis, Ascaris lumbricoides e Trichuris trichiura (Tabela 3).

A Tabela 4 mostra as relações entre as ligações de água e esgoto nas diversas regiões de Assis, como um índice de saneamento para a população atendida pelos PAS. Como pode ser observado existe uma relação direta entre o número de ligações de água e esgoto e um decréscimo na porcentagem de exames positivos dentro da amostra analisada. 


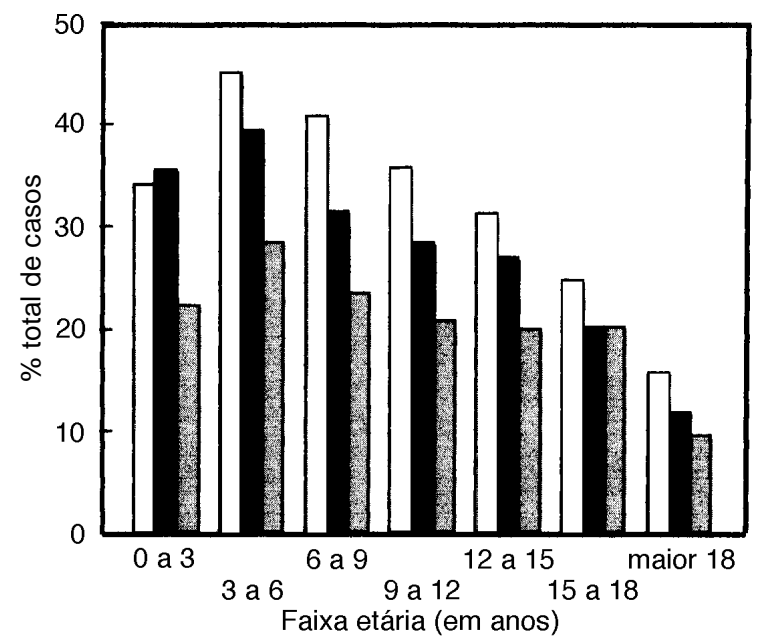

ㅁ1990 1991⿴囗十1992

Figura 5 - Distribuição por faixa etária de casos positivos de parasitos intestinais encontrados nos períodos estudados (19901991-1992).

Tabela 3 - Porcentagem de casos positivos de diferentes parasitos intestinais encontrados, por faixa etária, na amostra geral da cidade de Assis no período de 3 anos (1990/1991/1992).

\begin{tabular}{lcccccccccc}
\hline Faixa etária & Eh & $\mathrm{Gi}$ & $\mathrm{Al}$ & $\mathrm{Tt}$ & $\mathrm{A}$ & $\mathrm{Sm}$ & $\mathrm{Hn}$ & $\mathrm{Ev}$ & $\mathrm{T}$ & $\mathrm{Ss}$ \\
\hline $0-3$ & 0,2 & 18,4 & 8,6 & 2,3 & 0,4 & - & 0,8 & 0,04 & 0,04 & 0,8 \\
$3-6$ & 0,7 & 17,7 & 8,3 & 4,5 & 1,1 & - & 3,3 & 0,3 & - & 1,1 \\
$6-9$ & 0,5 & 11,8 & 8,3 & 4,4 & 1,4 & - & 4,3 & 0,6 & 0,1 & 1,3 \\
$9-12$ & 0,6 & 9,6 & 7,1 & 3,9 & 3,9 & 0,3 & 3,9 & 0,7 & 0,4 & 1,9 \\
$12-15$ & 0,1 & 7,0 & 7,0 & 4,2 & 4,2 & 0,3 & 2,1 & 0,9 & 0,2 & 2,1 \\
$15-18$ & 0,5 & 4,2 & 5,3 & 2,7 & 2,7 & 0,1 & 1,8 & 0,8 & 0,8 & 1,6 \\
$>18$ & 0,6 & 2,6 & 2,2 & 0,8 & 0,8 & 0,2 & 0,7 & 0,1 & 0,6 & 2,6 \\
\hline
\end{tabular}

Eh. Entamoeba histolytica; Gi. Giardia intestinalis; Al. Ascaris lumbricoides; Tt. Trichuris trichiura; A. ancylostomatidae; Sm. Schistosoma mansoni; Hn. Hymenolepis nana; Ev. Enterobius vermicularis; T. Taenia sp; Ss. Strongyloides stercoralis.

Tabela 4 - Relação entre o número de ligações de água e esgoto, população atendida e porcentagem de casos positivos de parasitos durante 0 ano de 1992.

\begin{tabular}{lcccc}
\hline PAS & № ligações de água e esgoto (a) & População (b) & a/b (\%) & Exames positivos (\%) \\
\hline Marialves & 2337 & 11288 & 21 & 34,2 \\
Progresso & 2621 & 10839 & 24 & 28,8 \\
Centro & 4225 & 15170 & 28 & 15,2 \\
Xavier & 3416 & 12202 & 28 & 24,6 \\
Fiúza & 3206 & 8548 & 37 & 16,1 \\
Bonfim & 3866 & 13923 & 28 & 12,6 \\
\hline
\end{tabular}

\section{DISCUSSÃO}

Alguns trabalhos têm relacionado a freqüência das parasitoses intestinais com alguns fatores ambientais, sócio-econômicos e condições de saneamento básico ${ }^{6} 8$.
Os enteroparasitos mais encontrados nas populações dos seis postos de atendimento sanitário (Tabela 1) foram, em ordem decrescente: Giardia intestinalis, Ascaris lumbricoides e 
Trichuris trichiura. Fazendo um paralelo com os resultados obtidos por diferentes autores podese observar que as freqüências dos parasitas Giardia intestinalis (8,7\%), Ascaris lumbricoides $(5,5 \%)$ e Trichuris trichiura $(2,4 \%)$, encontrados na cidade de Assis, como um todo, são mais baixos, relativamente àqueles verificados em outros levantamentos. Em levantamento por amostragem realizado na cidade de São $\mathrm{Paulo}^{8}$, os autores obtiveram freqüências de, respectivamente, $14,5 \% ; 16,4 \%$ e $12,6 \%$. Em um trabalho desenvolvido entre moradores na CECAP, distrito de Botucatu ${ }^{15}$, as freqüências obtidas para cada um desses parasitos foram, respectivamente, de: 9,7\%; 7,6\%; 17,3\%. Posteriormente, na mesma cidade de Botucatu ${ }^{4}$, um levantamento entre escolares de um região peri-urbana apresentou freqüências de 10\%; 11,5 \% e 16,4\%. Quando se considera os dados do PAS de Vila Marialves, que apresenta a maior prevalência de parasitoses e os piores indicadores sócio-econômicos entre as regiões consideradas no presente levantamento (Tabela 1) obtém-se freqüências de: $\mathbf{G}$. intestinalis (17\%), A. lumbricoides (13,1\%) e T. trichiura (5,9\%). Apreende-se desses dados que as freqüências de $G$. intestinalis nas regiões abrangidas pelo PAS de Vila Marialves superam àquelas obtidas nos levantamentos acima citados, inclusive aquela de $13 \%$ obtida numa área de ocupação por população de baixa renda do Município de Guarulhos (SP) ${ }^{2}$. A prevalência de T. trichiura é expressivamente menor que os demais levantamentos, podendo-se inferir a possibilidade desse parasito estar sendo laboratorialmente sub-diagnosticado. Considerando-se as formas de transmissão da $G$. intestinalis decorre, particularmente, da água contaminada é fundamental uma reavaliação do quadro atual das parasitoses, buscando-se a identificação das causas e possíveis focos de contaminação, responsáveis pela manutenção das parasitoses com freqüências tão elevadas.

Quanto à freqüência de enteroparasitos observadas em diferentes faixas etárias durante o período de estudo (Figura 5), constata-se, a partir do primeiro ano de vida, um aumento progressivo na freqüência de enteroparasitoses, sendo que na faixa de 3 a 6 anos obtém-se a freqüência mais elevada $(38,2 \%)$. Embora mantendo freqüências elevadas a partir do 6 anos observa-se uma tendência à queda progressiva das freqüências. Para os maiores de 18 anos as freqüências são relativamente baixas $(12,3 \%)$. Diferentes autores ${ }^{3}{ }^{14}$ realizaram trabalhos de estudo sobre a freqüência de enteroparasitoses em diferentes faixas etárias, determinando que a faixa etária com índices mais elevados é a de 5 a 12 anos, situação muito semelhante à verificada no presente estudo, no qual as maiores freqüências foram observadas de 3 a 12 anos. As crianças estão mais expostas à contaminação em função do desconhecimento dos princípios básicos de higiene e da maior exposição a partir do intenso contato com o solo, que funciona como um referencial lúdico em torno do qual desenvolvem uma série de jogos e folguedos. Postula-se para uma série de parasitos, particularmente para o A. lumbricoides, que o decréscimo na ocorrência com o passar da idade, ou seja, as baixas taxas de incidência e prevalência em adultos, estariam condicionadas não só a uma mudança de hábitos, mas também ao desenvolvimento de imunidade progressiva e duradoura contra tais parasitos ${ }^{10}$.

As freqüências mais elevadas de enteroparasitos, no presente estudo (Figura 3 e Tabela 3), foram observadas nas populações que utilizam os PAS da Vila Marialves $(45,1 \%)$, Vila Progresso $(28,6 \%)$ e CSIII-Vila Xavier $(37,2 \%)$, que são regiões periféricas da cidade e que abrangem as populações de nível sócioeconômico mais baixo, enquanto freqüências menores foram encontradas nas populações dos PAS do Bairro Bonfim (16,2\%) e CSI-Centro $(17,6 \%)$ que englobam as populações com um com melhor nível sócio-econômico. A relação entre nível sócio-econômico, condições de saneamento básico e ocorrência de parasitoses tem sido objeto de numerosos estudos ${ }^{11}{ }^{15}$, ressaltando-se um levantamento realizado no Município de São Paulo, no qual os autores verificaram uma diferença de até nove vezes na prevalência existente entre os extratos sócioeconômicos extremos da população ${ }^{11}$.

Os resultados decorrentes de Vila Fiúza (Figura 3 e Tabela 3), merecem uma análise particular. O bairro à época do levantamento estava em formação, com crescimento populacional acelerado. A construção de um novo conjunto habitacional popular naquela região atraiu uma população predominantemente de trabalhadores não especializados e/ou de baixa renda familiar. Apesar disso apresentou, no contexto geral da cidade, uma freqüência relativamente baixa de parasitoses intestinais $(19,1 \%)$, havendo inclusive a possibilidade de que algumas das parasitoses diagnosticados no PAS de Vila Fiúza poderiam, na verdade, ter sido contraídos no 
bairro de origem das famílias que agora passavam a ocupar o novo conjunto habitacional. Por outro lado o bairro implantava-se com uma infra-estrutura de saneamento básico inicial adequada, apresentando a melhor relação entre população e número de ligações de água e esgoto (Figura 4 e Tabela 4). O aumento na freqüência entre 1991 e 1992 poderia ser decorrente de um desvio em função da pequena amostra de exames realizados em 1992, direcionados, talvez, em função de queixas específicas e quadro clínico sugestivo de parasitose intestinal. Deve ser lembrado que o levantamento em questão não foi decorrente de uma busca ativa de casos, mas de exames parasitológicos realizados em pacientes que procuraram o PAS por diferentes motivos.

Em síntese, constata-se no período analisado uma tendência para queda na prevalência das parasitoses intestinais, o que coincide com 0 aumento das ligações de água e esgoto em todas as regiões da cidade de Assis (Tabelas 3 e 4). Evidencia-se portanto uma relação inversa entre a prevalência de exames positivos e a população atendida por ligações de água e esgoto. A partir da análise da Tabela 4 torna-se bastante evidente esta correlação. A maior freqüência de exames positivos é encontrada justamente no PAS de Vila Marialves, região apresentando as mais precárias condições de saneamento básico.

\section{AGRADECIMENTOS}

Agradecemos ao CNPq pelo apoio financeiro e ao Professor José Luis Guimarães do Departamento de Psicologia Experimental e do
Trabalho (UNESP-Assis, SP) pela valiosa contribuição no início deste trabalho.

\section{REFERÊNCIAS BIBLIOGRÁFICAS}

1. Cherter L, Cabeça M, Catapani WR. Parasitoses intestinais. Revista Brasileira de Medicina 51:126-132, 1995.

2. Chieffi PP, Waldman EA, Torres DMCV, Shimara R, Mizumoto LC, Silva AMA, Uehara M. Enteroparitoses no municípío de Guarulhos, SP, Brasil. 1. Prevalência de infecções entre escolares residentes no Bairro de Taboão, em junho de 1984. Revista do Instituto Adolfo Lutz 48:7580, 1988.

3. Chieffi PP, Waldman EA, Waldman CCS, Sakata EE, Gerbi LJ, Rocha AB, Aguiar PR. Aspectos epidemiológicos das enteroparasitoses no Estado de São Paulo, Brasil. Revista Paulista de Medicina 99:34-36, 1982.

4. Dórea RCC, Salata E, Padovani CR, Anjos GL. Control of parasitic infections among school children in the periurban area of Botucatu, São Paulo, Brazil. Revista da Sociedade Brasileira de Medicina Tropical 29:425-430, 1996.

5. Fanuchi JN, Chimentão S, Santos MI, Bueno JM. Contaminação da água e altos índices de giardíase. Jornal de Pediatria 56:117-119, 1984.

6. Giazzi JF, Martini AS, Buainain A, Mendonça CP, Belda Neto FM, Santos JL. Levantamento de protozoários e helmintos em crianças de um núcleo populacional de Araraquara. Revista Brasileira de Farmácia 43:49-52, 1982.

7. Hoffman NA, Pons JA, Janer JL. The sedimentation Concentration method in Schistosomiase mansoni, Puerto Rico. Journal of Public Health 9:283-291, 1934.

8. Martini AS, Rodrigues VC, Taba MRM, Fujimori C. Avaliação da presença de enteroparasitas em crianças de um centro de convivência infantil. Revista da Faculdade de Odontologia de Ribeirão Preto 22:17-20, 1985.

9. Monteiro CA, Chieffi PP, Benicio MHA, Dias RMS, Torres DMAGV, Mangini ACS. Estudo das condições de saúde das crianças do município de São Paulo (Brasil), 1984/ 1985. VII-Parasitoses intestinais. Revista de Saúde Publica 22:8-15, 1988.

10. Neves DP, Melo AL, Genaro O, Linardi PM. Parasitologia Humana, 9a edição, Atheneu, São Paulo, 1995.

11. Nussenzveig I, Natale A, Malheiro MEN, Malaco MML. Prevalência de anemia e de parasitoses intestinais em escolares do minicípio de São Paulo. Resultado do emprego da merenda escolar e de drogas antiparasitárias. Revista Paulista de Medicina 100:32-39, 1082.

12. Oliveira MR, Barbosa MA, Salata E, Sogayar MITL, Sogayar R, Correa FMA. Prevalência de enteroparasitoses na população urbana do $2^{\circ}$ Subdistrito de Botucatu. Revista de Saúde Publica 8:213-234, 1974.

13. Pedrazzani ES, Mello DA, Pizzigati CP, Pripas S, Fucci $M$, Santoro MCM. Helmintoses intestinais. III- Programa de Educação e Saúde em Verminose. Revista de Saúde Publica 23:189-195, 1989.

14. Pedrazzani ES, Mello DA, Pripas S, Fucci M, Barbosa CAA, Santoro MCM. Helmintoses intestinais. IIPrevalência e correlação com renda, tamanho da família, anemia e estado nutricional. Revista de Saúde Publica 22:384-389, 1988.

15. Salata E, Corrêa FMA, Sogayar R, Sogayar MIL, Barbosa MA. Inquérito parasitológico na Cecap.- Distrito-sede de Botucatu, Estado de São Paulo, Brasil. Revista de Saúde Publica 6:385-392, 1972. 
16. Sigulem DM, Tudisco ES, Paiva ER, Guerra CCC. Anemia nutricional e parasitose intestinal em menores de 5 anos. Revista Paulista de Medicina 103:308-312, 1985.
17. Vinha C, Martins MRS. Parasitoses intestinais entre escolares. Jornal de Pediatria 50:79-84, 1981. 